\title{
Diseño conceptual de sistema alternativo para la estabilización de taludes con neumáticos usados
}

\section{Conceptual design of alternative system for slope stabilization with used tires}

\author{
Manuel Isaac Pérez ${ }^{*}$, Emmanuel Jiménez, ${ }^{l}$, Manuel Arcia ${ }^{l}$ \\ ${ }^{I}$ Centro Regional de Panamá Oeste, Universidad Tecnológica de Panamá
}

\begin{abstract}
Resumen La corta vida útil de los neumáticos y su mala disposición resulta ser un problema para el medio ambiente. Este artículo propone un diseño conceptual para la retención de taludes con neumáticos usados, arbustos como medida de protección a la erosión y un drenaje francés para el desalojo del agua. Se derivan ecuaciones para definir la geometría del talud con el diseño propuesto y se realizaron ensayos para la caracterización de una muestra de suelo perteneciente a un talud en Capira, Panamá Oeste, siguiendo las normas ASTM. Adicionalmente, se realizó un ensayo a tracción con tiras de neumático usado y se hicieron modelos 3D en el software SkecthUp. Los resultados de esta investigación muestran el modelo 3D del muro propuesto, el suelo ensayado que corresponde a un limo elástico con arena; con humedad óptima de $33 \%$ y densidad máxima de 13,3 kN/m³ límite líquido y plástico de $63 \%$ y 33,7\% respectivamente, ángulo de fricción de $22^{\circ}$ y cohesión de $36,7 \mathrm{kPa}$. Con el ensayo a tracción se obtuvo un promedio a rotura de 12,55 kN. También se presenta un Windows Form App para el diseño geométrico del talud y se propone un ensayo a fricción como trabajo futuro.
\end{abstract}

Palabras clave Ensayos de suelos, ensayo a tracción, Microsoft Excel VBA programador, muro de retención.

\begin{abstract}
The short life of tires and their poor disposition turns out to be a problem for the environment. This article proposes a conceptual design for the retention of slopes with used tires, shrubs as a measure of protection against erosion and a French drain fort he evacuation of water. Equations are derived to define the geometry of the slope with the proposed design and tests were carried out for the characterization of a soil sample belonging to a slope in Capira, Panama Oeste, using ASTM standards. Additionally, a traction $\mathrm{s}$ test was performed with used tire strips and 3D models were made in the SkecthUp software. The results of this investigation show the 3D model of the proposed wall, the tested soil which corresponds to an elastic silt with sand; with optimum moisture content of $33 \%$ and maximum density $13,3 \mathrm{kN} / \mathrm{m}^{3}$, liquid and plastic limit $63 \%$ and $33,7 \%$ respectively, friction angle 22 ${ }^{\circ}$ and cohesion $36,7 \mathrm{kPa}$. With the tensile test a breaking average 12,55 kN was obtained. A Windows Form App for the geometric design of the slope is also presented and a friction test is proposed as future work.
\end{abstract}

Keywords Soil test, tensile strength test, Microsoft Excel VBA programming, retaining wall.

* Corresponding author: manuel.perez1@utp.ac.pa

\section{Introducción}

El aumento en la tenencia de autos y la necesidad de reemplazar los neumáticos, una vez cumplen su vida útil, resulta en un problema medio ambiental por la quema de este material y su mala disposición. Según Research and Markets, está proyectado un crecimiento global en el mercado de los neumáticos de 731,6 millones de unidades [1]. En el caso de Panamá, al relleno sanitario de Cerro Patacón llegan 1,5 toneladas de llantas por día [2]. Dado este problema: ¿Cómo se puede aprovechar este material una vez cumplida su vida útil? A esta pregunta Long, et al. (1993) encontraron que podían utilizar los neumáticos como una estructura para estabilizar taludes, por lo cual, concretizaron su idea con un muro de cinco metros de altitud y 10 metros de longitud al que denominaron Pneusol [3]. Sayão et. al., también construyeron un muro Pneusol con capas de llantas rellenas con suelo compactado de cuatro metros de altitud y 60 metros de largo.

Terre Armee con más de 50 años de experiencia en el campo de la geotecnia comercializa su producto Reinforced Earth ${ }^{\circledR}$, que consiste en una estructura de retención a gravedad con refuerzos de acero o geosintéticos trabajando a tracción con un relleno granular compactado, el cual no requiere equipo pesado para su construcción y resiste cargas vivas y muertas impuestas por estructuras, vehículos y equipos pesados [4]. 
El caucho sintético con mayor demanda a nivel mundial es el Estireno Butadieno (SBR), debido a que se utiliza principalmente en la fabricación de neumáticos [5].

El objetivo de esta investigación es proponer un diseño de muro de retención con taludes de neumáticos usados y hacer un estudio inicial de caracterización de suelo, para que en una segunda etapa se realice un ensayo a fricción y el análisis del sistema en analogía al análisis para muros mecánicamente reforzados; además presentar un Windows Form App de Microsoft Excel para el definir la geometría del muro con el diseño propuesto. Alineado así, con el noveno objetivo de desarrollo sostenible (ODS) del Programa de las Naciones Unidas [6].

En este artículo se presenta primeramente un estudio de muro de retención cuyo diseño fue construido. Luego, se presenta la metodología desarrollada para evaluar en un Windows Forms App el diseño propuesto para un talud específico, las normas ASTM utilizadas para los ensayos de suelo y tira de neumático; así como, la definición de un ensayo a fricción para una segunda etapa de este estudio. Después, se describe el diseño de muro de retención propuesto, donde se incluye un sistema de drenaje y control de erosión, y se muestra el modelo 3D del muro. Seguido, se muestran los resultados de los ensayos y se describe su significado; además se discute el ensayo a fricción de la segunda etapa y su dependencia de los ensayos desarrollados en este estudio. También, se discute sobre los hallazgos y metodologías utilizadas en otras investigaciones de muros con neumáticos. Finalmente se presentan las conclusiones y agradecimientos.

\section{Muro con neumáticos}

Estos sistemas, en comparación a otros, evitan la contaminación y la proliferación de enfermedades a causa de los mosquitos. En la figura 1 se muestra el arreglo de un muro de retención por capas.

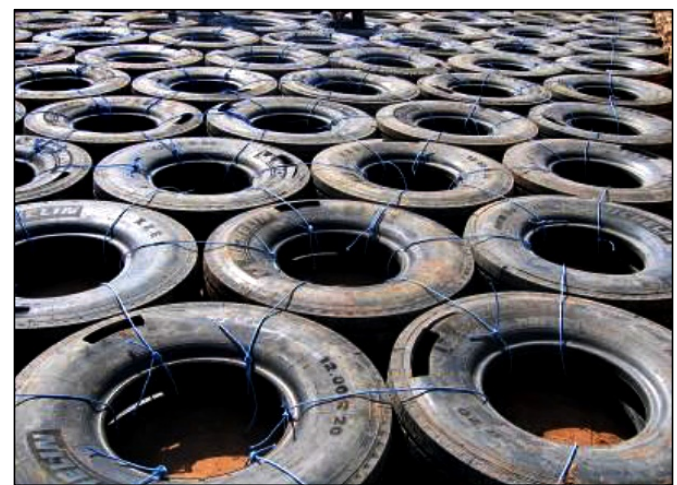

Figura 1. Arreglo en la capa de los neumáticos.

Fuente: Energy Procedia. Belabdelouhab et al. (2014).

Por no requerir mano de obra calificada y la abundancia de este material, es de fácil construcción; además, las características del material de los neumáticos tienen una alta duración [7].
Belabdelouhab et al. (2014) estudiaron un muro de 20 metros de alto y 100 metros de longitud con neumáticos de un diámetro externo y grosor de 1,20 y 0,33 metros, respectivamente [8]. El arreglo de los neumáticos en este estudio es el que se muestra en la figura 1.

\section{Métodos y materiales}

En la figura 2 se muestra un diagrama de flujo de la metodología desarrollada en este artículo.

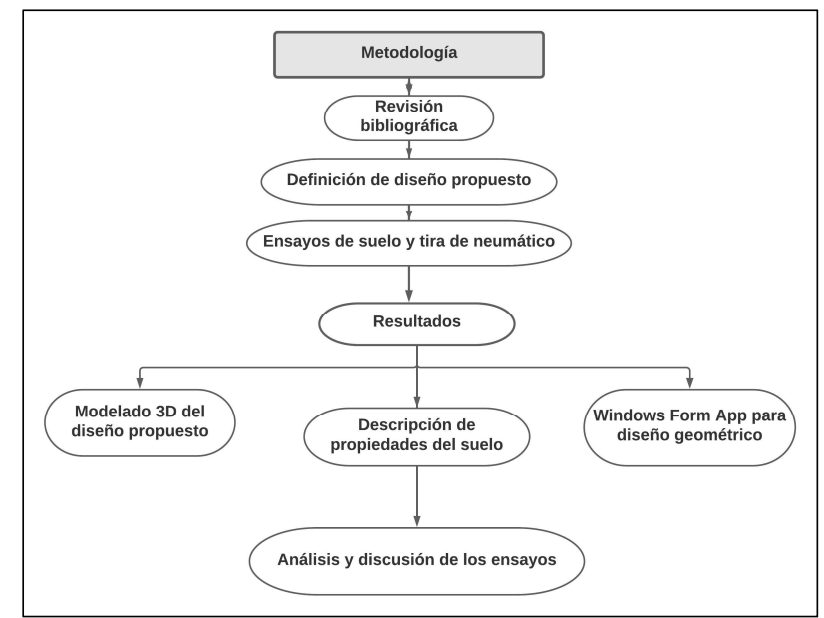

Figura 2. Diagrama de flujo de la metodología.

Fuente: Los autores.

\subsection{Definición del diseño}

En base a la revisión de la bibliografía y la aplicación de neumáticos usados en el sistema, se definió una geometría de diseño. Esta geometría propuesta se enmarcó en un Windows Form App por medio de la interfaz programador Visual Basic de software Microsoft Excel.

La geometría del muro con el diseño propuesto depende del ancho y diámetro externo del neumático, así como la separación de sesgado entre neumáticos. Se realizó un diagrama de cuerpo libre de la parte frontal del sistema propuesto y se le asignaron letras a las variables que determinan su geometría.

Las variables de la figura 3 representan las siguientes características:

- P: diámetro exterior de neumático (m).

- Q: ancho de llanta (m).

- $\Delta x$ : sesgo horizontal entre neumáticos (m).

- $\sigma$ : ángulo del talud (grados).

- Lh: longitud horizontal (m)

- Lv: longitud vertical (m).

- $\mathrm{n}$ : cantidad de unidades llanta centrales (unidades).

- E: unidades de neumático uno sobre otro (unidades). 
Pérez (et al): Diseño conceptual de sistema alternativo para la estabilización de taludes con neumáticos usados

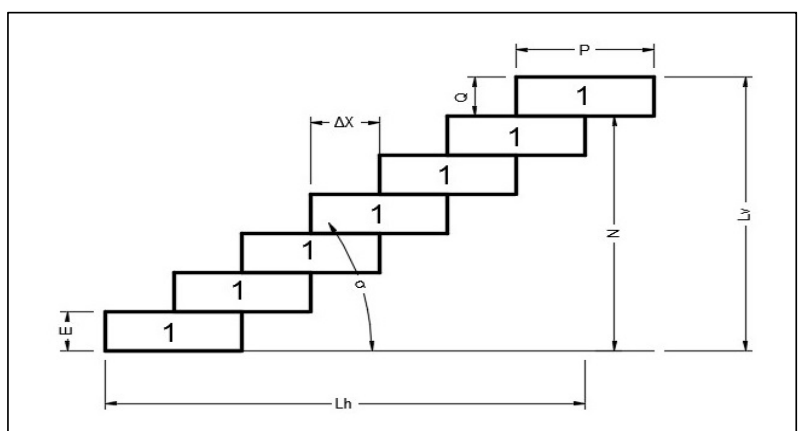

Figura 3. Diagrama de cuerpo libre y variables del sistema.

Fuente: Los autores.

Del análisis de variables, se derivaron dos conjuntos de ecuaciones. Las columnas de tres ecuaciones que se presenta a continuación corresponden a los dos conjuntos de ecuaciones que se derivaron:

$$
\begin{array}{cc}
L h=\frac{L v}{\tan \sigma} & E=\frac{(p / 2)(\tan \sigma)}{Q} \\
n=\frac{2 L h}{p}-2 & n=\frac{L v}{E Q}-2 \\
E=\frac{(p / 2)(\tan \sigma)}{Q} & L h=p+\frac{n p}{2}
\end{array}
$$

Estas ecuaciones se cargaron al Windows Forms App donde se solicitan los siguientes datos de entrada: longitud, ángulo y altura del talud, diámetro exterior y ancho del neumático. Como resultado, el formulario imprime el valor de Lh en metros, la cantidad de neumáticos en unidad-neumático $\mathrm{y}$ el valor de $\mathrm{n}$ que corresponde a la cantidad de unidadneumático centrales en el sistema. Además, proporciona los errores en el ángulo y la longitud vertical (Lv) del talud y la cantidad total de neumáticos necesarios para su construcción.

Con ayuda del software SkecthUp se confeccionó el modelo 3D del diseño propuesto con un $\Delta \mathrm{x}=\mathrm{p} / 2$.

\subsection{Ensayos de suelo}

Para los ensayos de suelo se tomó una muestra proveniente de un sitio de deslizamiento en el área de Capira, Panamá Oeste.

Cardoso (2014) realizó ensayos de suelo como los que se presentan en este estudio. Como datos de entrada para el modelado numérico de un muro Pneusol, y encontró que mientras se ocupe con tierra los espacios entre los neumáticos, una geometría del muro en pendiente le da a la estructura una marcada rigidez [9].

La figura 4 muestra el ensayo de límites de Atterberg (ASTM D 4318), mientras que la figura 5 presenta el análisis granulométrico siguiendo la norma ASTM D 6913, y la figura 6 ilustra el ensayo de corte directo.

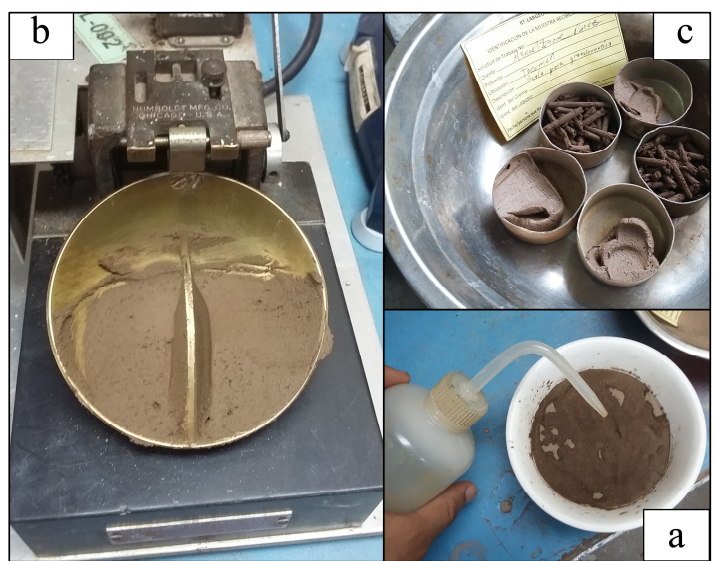

Figura 4. a) Humedecimiento de la muestra de suelo. b) Copa de Casagrande para determinar límite líquido. c) Muestras de suelo luego de 24 horas al horno. Fuente: Los autores.

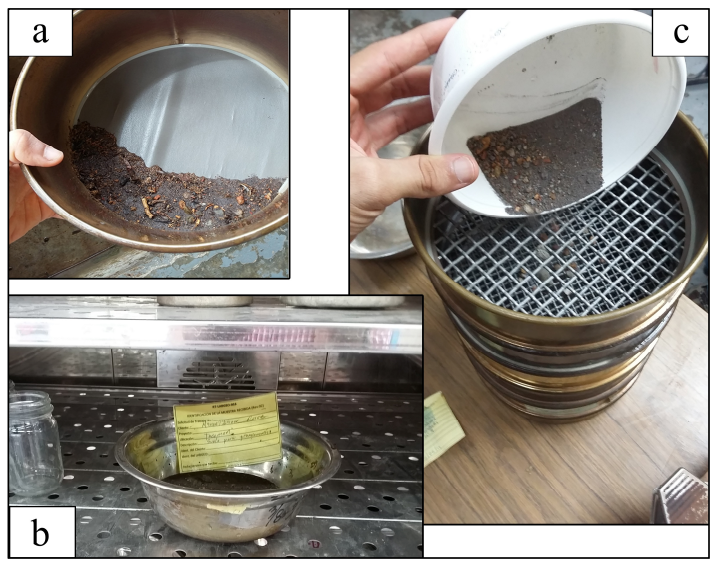

Figura 5. a) Lavado de muestra de suelo. b) Colocación de la muestra en el horno por 24 horas. c) Tamizaje manual con muestra de suelo seca. Fuente: Los autores.

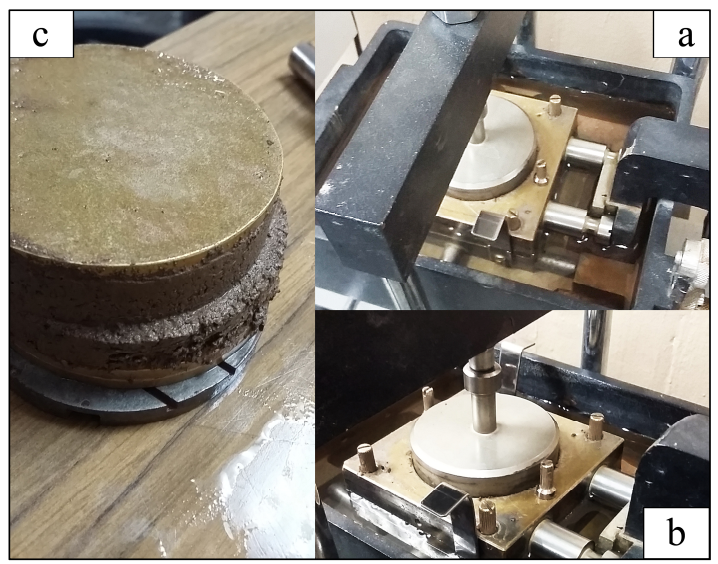

Figura 6. a) Inicio del ensayo en el equipo ELE. b) Continuación del ensayo minutos después. c) Muestra de suelo una vez finalizado el ensayo.

Fuente: Los autores.

El ensayo de corte directo (ASTM D 3080) se realizó en un equipo de corte directo/residual ELE en condiciones drenadas y consolidadas con cargas normales aplicadas de 1,5 y $10 \mathrm{~kg}$. 
Con este ensayo se obtiene el ángulo de fricción y la cohesión del suelo; ambos parámetros importantes en el análisis geotécnico.

Se llevó a cabo el ensayo de Proctor estándar de acuerdo con la norma ASTM D 638 para determinar la densidad máxima del suelo y la humedad óptima. En la figura 7 se ilustra cuando se retira parte del exceso de suelo en el equipo.

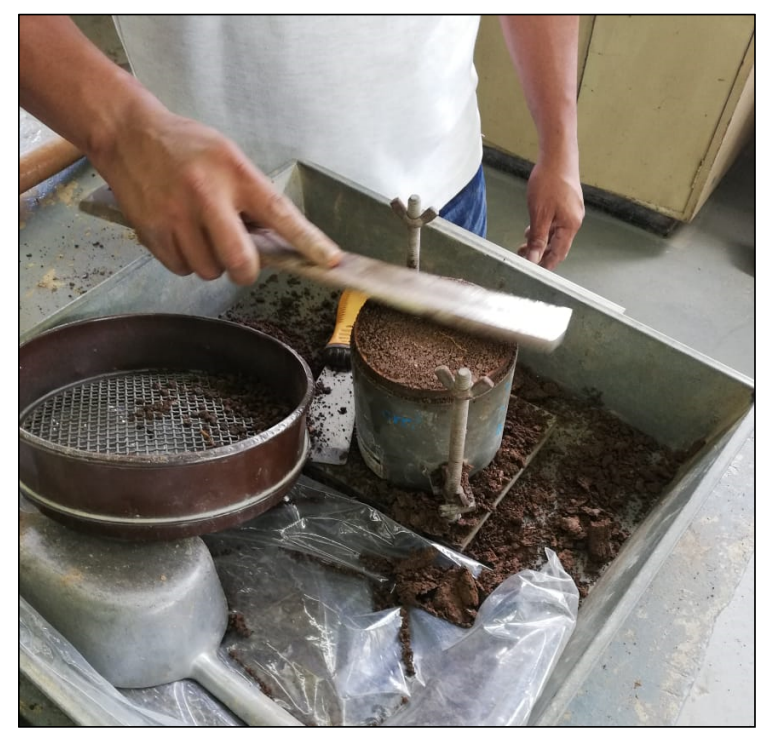

Figura 7. Ensayo de Proctor estándar.

Fuente: Los autores.

\subsection{Ensayo para tira de neumático}

Para el ensayo de tensión se prepararon tres probetas (de llantas usadas) de acuerdo a la norma ASTM A370. Con una máquina de prueba universal se ensayó hasta la rotura y se calculó el promedio de la fuerza aplicada. En la figura 8 se muestra el proceso realizado.

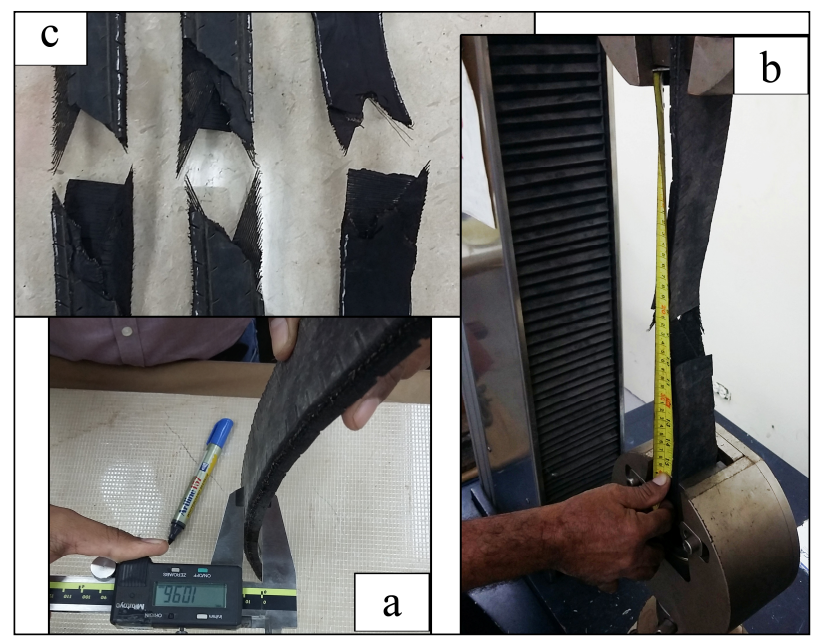

Figura 8. a) Medición de dimensiones de la probeta con pie de rey digital. b) Tira de neumático en la máquina de prueba universal. c) Fractura de las tres probetas de ensayo de neumáticos usados.

Fuente: Los autores.

\subsection{Planteamiento de trabajo futuro}

En base a la revisión de literatura se plantea un prototipo para el ensayo a fricción, el cual es modelado en el software SkecthUp.

\section{Resultados y discusión}

\subsection{Diseño propuesto}

La estructura de retención que se propone consta de una unidad-neumático que se organiza una sobre otra a medio diámetro de separación. En un mismo nivel se colocan una al lado de la otra; para el siguiente nivel van sesgadas a medio diámetro, como el arreglo en el que están organizados los bloques en las paredes. La unidad-neumático puede estar constituida de uno o varios neumáticos ajustados perfectamente uno sobre otro. Como resultado, la parte frontal del muro tendrá una organización tipo escalera. Su parte posterior estará conformada por tiras de neumático fijadas al arreglo tipo escalera; estas actuarán como elementos a tracción para resistir la fuerza de empuje del talud. En las soluciones comerciales actuales como las de Terre Armee esta función es realizada por geosintéticos poliméricos. Estas estructuras de suelo reforzado también se conocen con el nombre de muros mecánicamente reforzados (MSE). El interior de los neumáticos del primer nivel, estarán empotrados en el suelo, y se rellenarán con mortero. En los demás niveles se rellenan con tierra compactada y en su parte frontal se le colocan llorones para el mejor drenaje.

La compañía Geomatrix, fabricante de una gran variedad de geosintéticos explica que: "Este mecanismo de transferencia ocurre principalmente en los geotextiles. Cuando se da un desplazamiento cortante relativo entre el geotextil y las partículas del suelo, las cargas normales al plano del geotextil generan fuerzas de fricción en toda la superficie, las cuales se integran para generar una fuerza que se opone al arrancamiento de este del interior de la masa de suelo" [10]. En la figura 9 se bosqueja la transferencia de estos esfuerzos.

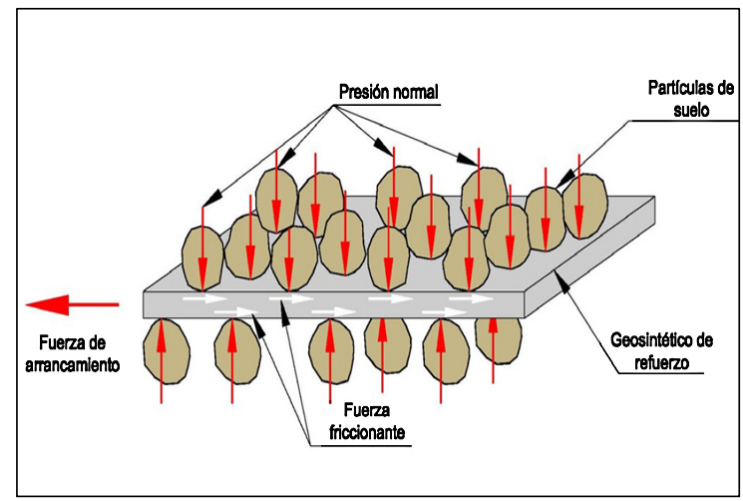

Figura 9. Transferencia de esfuerzos de fricción entre el suelo y el geosintético.

Fuente: Geomatrix [10]. 
En la investigación de Barro et al. (2019) se propuso un sistema de retención con llantas dispuestas verticalmente con refuerzo de geomallas en lugar de tiras de neumáticos como se propone en este estudio [11].

Las intensas precipitaciones sobre el suelo desnudo de los neumáticos comprometen la estabilidad del muro; este debe mantener su estructura intacta para ejercer su función. Para ello, las herbáceas y arbustos de rápido crecimiento son una buena alternativa para darle protección a los taludes contra la erosión, debido a la gran cantidad de raíces que profundizan en el suelo buscando la humedad. El Rosmarinus officinalis Prostratus (romero rastrero) y Lavandula angustifolia (lavanda) entran dentro de esta categoría [12,13]. También se puede optar por la Salvia rosmarinus (romero común). En la figura 10 se muestra un plantón joven. Como se ve en la figura $10 \mathrm{~b}$ las raíces de esta planta son voluminosas y pequeñas, amarrando el suelo e impidiendo la erosión.

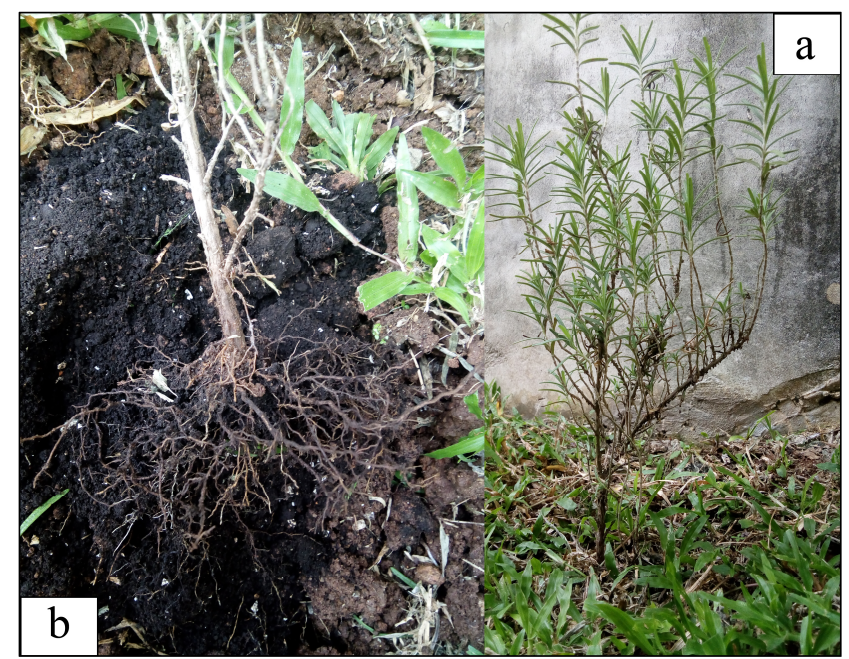

Figura 10. Romero común.

Fuente: Los autores.

Durante las intensas precipitaciones, el muro también debe cargar con la presión hidrostática, la cual se debe evacuar para evitar fallas. En este sentido, se plantea el uso de un dren francés con orificios de $5 \mathrm{~cm}$ a $22.5^{\circ}$ y $45^{\circ}$ respecto a la vertical y un diámetro de tubería de seis pulgadas con pendiente de $2 \%$. Para el mejor desarrollo de su función las tuberías de PVC se envuelven con un material permeable (geotextil no tejido) que le permitirá filtrar el agua, de manera que no se laven las partículas de suelo fino. Esto también evita que granos de mayor tamaño tapen los orificios del dren.

De acuerdo a Ditchter \& Neira [14] 1 de cada tres panameños entrevistado con auto tiene un Toyota. Siendo el modelo Corolla uno de los tres vehículos más vendidos en todo el mundo [15] cuyo modelo de neumáticos es el 205/55R 16 [16]. Se recomienda el uso de estos neumáticos para que conformen la estructura del muro; la cual debe estar constituida de neumáticos con un tamaño lo más uniforme posible.
En la figura 11 se muestra el modelo 3D del diseño propuesto para el muro de retención.

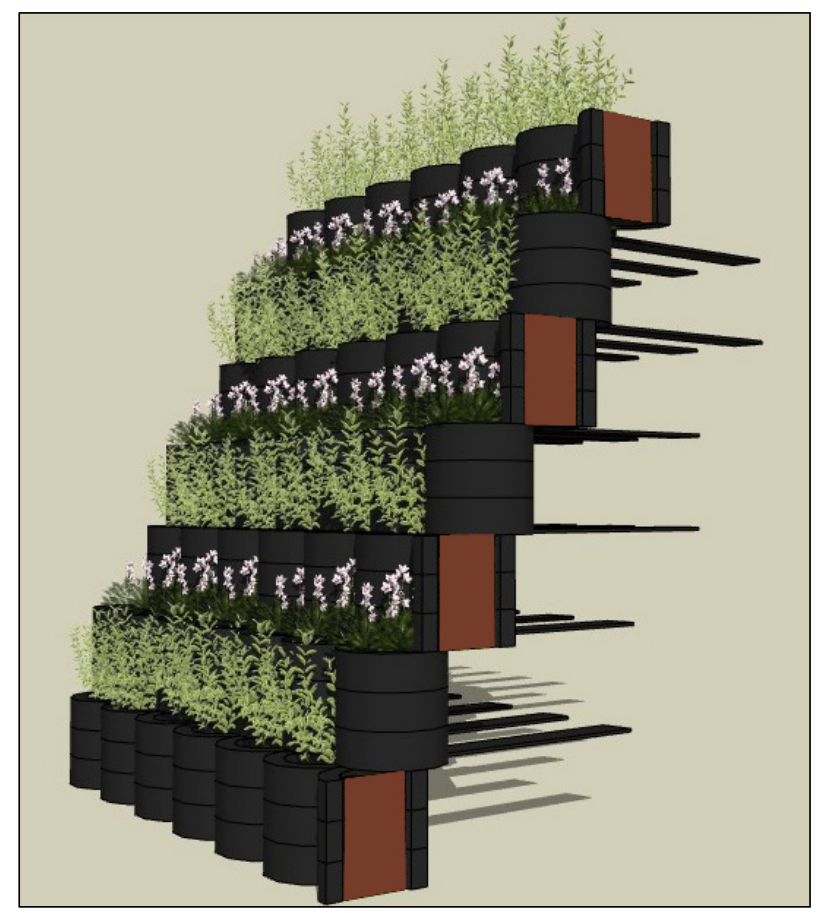

Figura 11. Modelo 3D del diseño propuesto.

Fuente: Los autores.

\subsection{Resultados de los ensayos}

En la figura 12, para los veinticinco golpes de la Copa de Casagrande, le corresponde un valor de $63 \%$ de contenido de agua. Esto quiere decir, que la muestra de suelo pierde toda su resistencia al corte y puede fluir como un líquido a partir de un contenido de agua superior al $63 \%$.

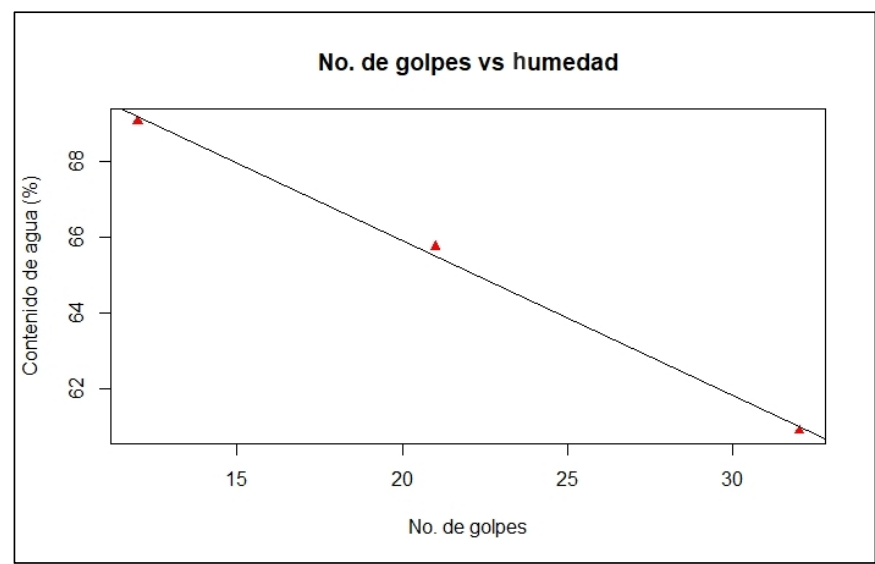

Figura 12. Gráfico para ensayo de límites de Atterberg, límite líquido (LL). Fuente: Los autores.

La figura 13 muestra el ensayo a tracción con tiras de neumático. 


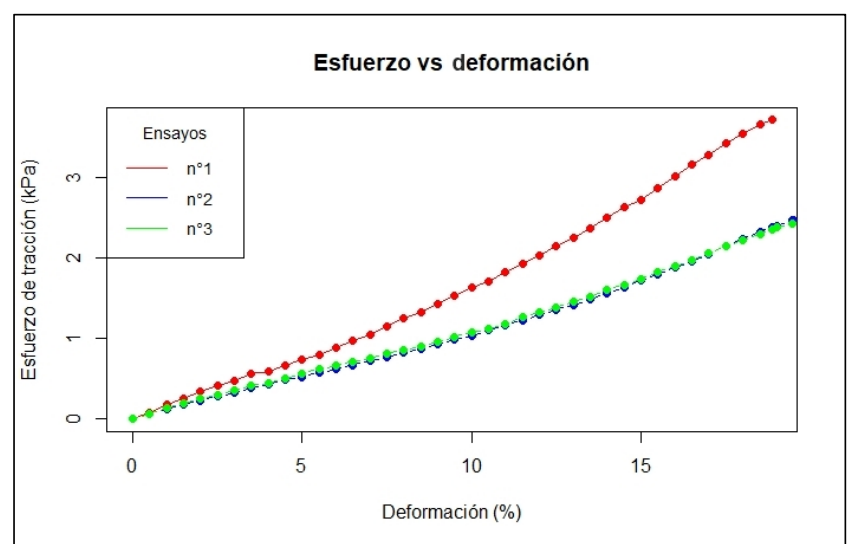

Figura 13. Gráfico de esfuerzo vs deformación para ensayo de tira de neumáticos usados.

Fuente: Los autores.

En la figura $8 \mathrm{c}$ se muestra que las tiras de neumático presentaron el mismo patrón de corte al romperse, con una fuerza promedio de 12,55 $\mathrm{kN} \pm 0,20 \mathrm{kN}$. El ensayo 1 de tracción mostró un comportamiento diferente a los demás, como se muestra en la figura 13; esto a causa de un menor grosor en la longitud de calibre en esta probeta.

En un ensayo a tracción realizado por Bellas (2012) con caucho estireno-butadieno (SBR) puro se encontró un esfuerzo a rotura de 1,76 $\pm 0,08 \mathrm{MPa}$ [17], mientras que, en el presentado

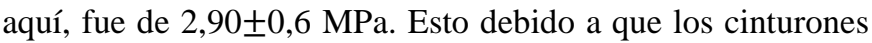
estabilizadores de acero presentes en el neumático le añaden resistencia a diferencia del SBR puro ensayado por Bellas.

En la tabla 1 se muestra el compendio de los resultados obtenidos de los ensayos y la norma ASTM respectiva.

Tabla 1. Resultados generales de los ensayos

\begin{tabular}{|c|c|c|c|c|c|}
\hline \multirow{2}{*}{\multicolumn{2}{|c|}{ Tipo de ensayo }} & \multirow{2}{*}{ Norma } & \multicolumn{2}{|c|}{ Resultados } & \multirow{2}{*}{ Comentarios } \\
\hline & & & Magnitud & Unidad & \\
\hline \multicolumn{2}{|c|}{ tensión } & ASTM A 370 & 12,55 & $\mathrm{kN}$ & $\begin{array}{c}\text { promedio a rotura de probetas } \\
\text { de llantas usadas }\end{array}$ \\
\hline \multirow{3}{*}{ Atterberg } & LL & \multirow{3}{*}{ ASTM D 4318} & 63 & $\%$ & \multirow{3}{*}{ límites de Atterberg } \\
\hline & LP & & 33,7 & $\%$ & \\
\hline & IP & & 29,3 & $\%$ & \\
\hline \multirow{2}{*}{ corte directo } & $\Phi$ & \multirow{2}{*}{ ASTM D 3080} & 22 & grados & \multirow{2}{*}{ equipo corte/residual ELE } \\
\hline & $\mathrm{c}$ & & 36,7 & $\mathrm{kPa}$ & \\
\hline \multicolumn{2}{|c|}{ granulometría } & ASTM D 6913 & \multicolumn{2}{|c|}{$\begin{array}{c}\text { limoso elástico con arena } \\
(\mathrm{MH})\end{array}$} & 7.5 YR 3/3 (Munsell) \\
\hline \multirow{2}{*}{ compactación } & $\gamma \mathrm{d}$ & \multirow{2}{*}{ ASTM D 698} & 13,30 & $\mathrm{kN} / \mathrm{m}^{3}$ & densidad seca máxima \\
\hline & $\mathrm{w}$ & & 33 & $\%$ & humedad óptima \\
\hline
\end{tabular}

Fuente: Los autores.

Con el ensayo de corte directo se obtuvo el valor de $\Phi$ (ángulo de fricción), con el cual se puede determinar la cuña de falla de la muestra de suelo estudiada.

Como consecuencia de la presencia de finos en la composición del suelo con un valor de $84,3 \%$ del total de la muestra ensayada, se expresa un valor para la cohesión de 36,7 $\mathrm{kPa}$.

El ensayo de compactación proporciona la máxima densidad del suelo frente a una humedad óptima; es decir, que el suelo estudiado requiere de un contenido de agua del $33 \%$ para que se reduzcan los espacios vacíos, al mismo tiempo que mantiene su consistencia. Si se sigue aumentando el contenido de agua, este perderá su consistencia y al sobrepasar el límite líquido, empezará a fluir.

Además, se identificó el color de la muestra de suelo como chocolate oscuro por medio de la tabla de Munsell, tal como se describe en la columna de comentarios de la tabla1.

Para el análisis de fuerzas del muro, con el ensayo a tracción se tiene una referencia de hasta qué punto, por sí solo, la tira de neumático falla a tracción.

\subsection{Windows Form App}

El Windows Form App de Microsoft Excel que se presenta en la figura 14, realiza los cálculos para definir la geometría del muro con un valor de $\Delta \mathrm{x}=\mathrm{p} / 2$. También muestra el porcentaje de error para el ángulo y altura de los valores de entrada, para los dos conjuntos de ecuaciones derivadas.

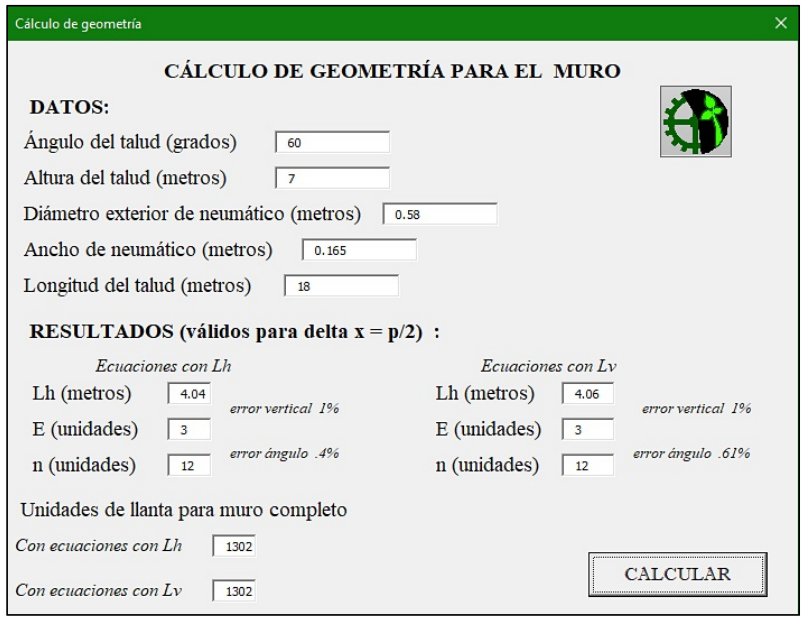

Figura 14. Windows Forms App de Microsoft Excel para el cálculo de geometría del muro de retención.

Fuente: Los autores.

\subsection{Trabajo futuro propuesto}

Para una segunda etapa de esta investigación se plantea realizar un ensayo a fricción; este consiste en cortar una tira de neumático usada en su circunferencia y colocarla longitudinalmente entre un suelo compactado al 95\% Proctor estándar dentro de una caja de $62 \mathrm{~cm}$ de altura. Luego, se le aplica una fuerza de tensión en uno de sus extremos para intentar sacar la tira de la interfaz suelo-suelo mientras se registran los desplazamientos con un deformímetro digital.

Este ensayo se puede realizar con la densidad máxima, debido a que se conoce del ensayo de compactación el contenido de agua necesario para ello. Además, conocida la cuña de falla, se puede determinar la longitud efectiva de la tira de neumático que estará actuando a fricción tanto en su parte superior e inferior, fuera de la superficie de falla; y como se conocen las propiedades mecánicas del suelo con los demás ensayos, se podrá determinar experimentalmente la fuerza resistente a la zafadura o fuerza de arrancamiento. Este valor representará un valor mínimo, pues a medida que se avance en 
profundidad aumentará la presión, por tanto, también la fuerza resistente a la zafadura $[18,19,20]$.

Se ilustra en la figura 15 el prototipo para el ensayo propuesto.

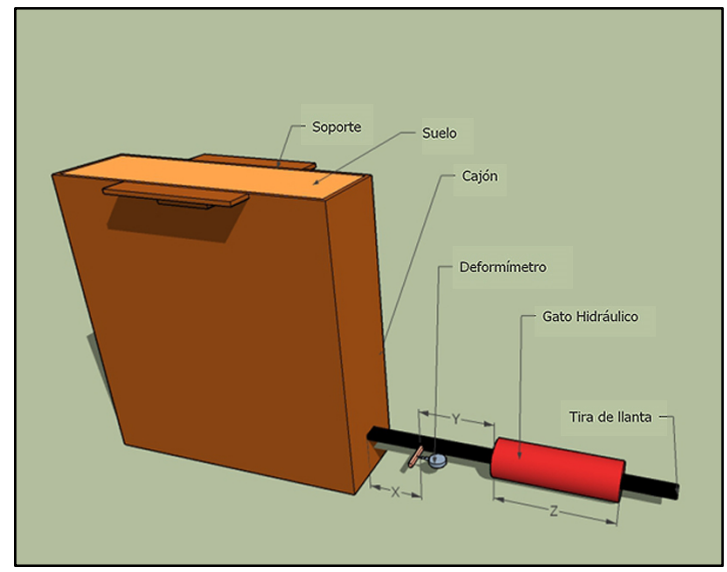

Figura 15. Prototipo para ensayar a fricción tira de neumático. Fuente: Los autores.

El suelo que se ha ensayado contiene una mayor proporción de finos, por lo que el aporte de fricción no será tan alto si se compara con un suelo granular. Sin embargo, los resultados de este ensayo, en la condición más desfavorable, son la base para el cálculo y diseño del muro en retención en analogía con el método utilizado para el diseño de un MSE [20], donde se utilizan tiras acero, en lugar de las tiras de neumáticos propuestas en este estudio.

Otros autores han diseñado y construido muros de retención con neumáticos, incluso han monitoreado sus desplazamientos con instrumentación de campo como lo presenta Medeiros et al. (2009) en su estudio; utilizaron inclinómetros y extensómetros magnéticos. Con este equipo se puede verificar la inclinación del muro y la deformación vertical [21]. También se pueden instalar piezómetros para verificar el nivel del agua en la parte posterior del talud. La principal causa de fallas en muros de retención es debido a la saturación del suelo, por lo cual, verificar la funcionalidad del sistema de drenaje es de vital importancia.

El material que une los neumáticos debe proporcionar resistencia a la corrosión y a las fuerzas cortantes. En la bibliografía consultada el material más utilizado es cuerda de polipropileno [11, 22]. Sin embargo, también se debe evaluar la unión entre la tira de neumático y el neumático de la parte frontal.

En esta propuesta se presenta el neumático completo como parte de la estructura frontal rellena con suelo en los niveles superiores y con mortero en el primer nivel. Sayão et al. (2002) encontraron que cortando la banda lateral del neumático la densidad aumentaba en $0,8 \mathrm{kN} / \mathrm{m}^{3}$ reduciendo así su comprensibilidad [23].

En un estudio realizado por Teixeira (2012), por medio del método de Bishop modificado, se determinó que el factor de seguridad global de un muro realizado por Sayão fue de 2,26 [24].

Con el apoyo de softwares para el análisis de esta propuesta de diseño como lo han hecho otros autores, se revelarían las fortalezas y debilidades del sistema propuesto para desarrollar una estructura que sea segura y confiable.

\section{Conclusiones}

El Windows Forms App que se desarrolló, permitirá evaluar rápidamente la posibilidad de utilizar el diseño propuesto en un sitio o si es necesario hacer algunas modificaciones en los extremos para cumplir la relación geométrica necesaria para el talud.

La segunda etapa de este estudio como trabajo futuro permitirá obtener un mejor conocimiento sobre la funcionalidad del diseño propuesto.

\section{AGRADECIMIENTOS}

A la Doctoranda Marta Cedeño por su apoyo para las gestiones del proyecto dentro del centro regional, a la Dra. Indra Candanedo por el apoyo financiero. Por otro lado, al Centro Experimental de Ingeniería (CEI) gestión del Lic. Jesús Villar e Ing. Carlos Lezcano, a los Ingenieros Gustavo Grimaldo, Josué Pazmino, especialmente al técnico Jairo Aguilar y al Ing. Amador Hassell por sus consejos para la segunda etapa de esta investigación.

\section{REFERENCIAS}

[1] GlobeNewswire. (2020) Growth Opportunities in the Tires Industry, 2020-2025 - Radialization Continues to Bulldoze Growth in Tire Markets of Emerging Countries. [Online]. Available at: https://www.globenewswire.com/newsrelease/2020/04/03/2011287/0/en/Growth-Opportunities-in-theTires-Industry-2020-2025-Radialization-Continues-to-BulldozeGrowth-in-Tire-Markets-of-Emerging-Countries.html

[2] Pérez K. (2019) Montaña de llantas en Cerro Patacón genera preocupación. TVN Noticias. [Online]. Available at: https://www.tvn-2.com/nacionales/Montana-llantas-CerroPatacon-preocupacion_0_5238226208.html

[3] Long N., Pierre L. (1993). "Le Pneusol: Recherches - Réalisations - Perpectives". Devantl'institut national des sciences appliquees de Lion. París, France.

[4] Terre Armee. "Rainforced Earth". [Online]. Available at: https://www.terre-armee.com/reinforced-earth/reinforced-earthmechnically-stabilized-earth/

[5] Castro G. (2008). "Materiales y Compuestos para la Industria del Neumático." Universidad de Buenos Aires [Online]. Available at: https://campus.fi.uba.ar/file.php/295/Material_Complementario/ Materiales_y_Compuestos_para_la_Industria_del_Neumatico.pd f [20 Apr. 2019]

[6] PNUD. "Objetivo 9: Industria, innovación, infraestructura." [Online]. Available at: http://www.pa.undp.org/content/panama/es/home/post-2015/sdgoverview/goal-9.html 
[7] Barón J. (2014). "Estabilización de Taludes por Medio de Muros de Llantas en el Barrio la Capilla Soacha Cundinamarca." Universidad Católica de Colombia, Colombia.

[8] Belabdelouhab F., Kebaïli N. (2015). "Large Scale Experimentation Slope Stability of «Soil Tyre» in Mostaganem (Algeria)". Energy Procedia, 74,699 - 706. doi:https://doi.org/10.1016/j.egypro.2015.07.805.

[9] Cardoso A. (2014). "Estruturas de Suporte de Terras Executadas com Pneus - Modelo à Escala Reduzida". Instituto Politécnico do Porto. Porto, Portugal.

[10] Geomatrix. "Conceptos Básicos para Estructuras de Suelo Reforzado." [Online]. Available at: http://gtech.geomatrix.co/uploads/1472242066_Guia_diseo_estructuras _MSE.pdf

[11] Barro P., Sarabia G., et al. (2019). "Retaining Wall based on mechanically stabilized tire stack". Revista ingeniería de construcción, 34(3), 252-267. https://dx.doi.org/10.4067/S071850732019000300252

[12] Bravo A. (2018) "Cómo afianzar un talud con plantas." Verde es Vida, 84, 52-53.

[13] Jardinería Panamá. "Lavanda" [Online]. Available at: https://vivero.jardineriapanama.com/products/lavanda?_pos=1\& sid=105df09f0\&_ss=r

[14] Ditchter \& Neira. "La posesión actual y compra futura de autos en Panamá." [Online]. Available at: http://www.dichterneira.com/wp-content/uploads/2016/02/20.-Insider_PA_Autom\%C3\%B3viles.pdf

[15] Panamá América. "Ricardo Pérez S.A. lidera venta de autos en Panamá." [Online]. Available at: https://www.panamaamerica.com.pa/economia/ricardo-perez-salidera-venta-de-autos-en-panama-1119785

[16] Ricardo Pérez S.A. "Corolla." [Online]. Available at: https://www.toyotarp.com/corolla/especificaciones

[17] Bellas R. (2012). "Formulación y Caracterización de Materiales Compuestos Integrados por una Matriz de Caucho EstirenoButadieno (SBR) Reforzada con Nanoarcilla" Universidad Da Coruña, España.

[18] Garnica P., Reyes R., Gómez J. (2013). "Diseño de muros reforzados con geosintéticos". Instituto Mexicano del Transporte. México. ISSN 0188-7297.

[19] Suarez J. (2012). "Diseño de Muros de Suelo Reforzado con Geosintético". Universidad Industrial de Santander, Bucaramanga, Colombia.

[20] Gunaratne M. The Foundation Engineering Handbook. Bosa Roca, United States: Taylor \& Francis Inc, 2006, pp. 450-482.

[21] Medeiros L., Garga V., et al. (2009). "Analysis of the Instrumentation of a Scrap Tire Reinforced Retaining Wall" presented at 11th Panamerican Conference on Soil Mechanics and Geotechnical Engineering, 1, 19-25.

[22] Escobar W. (2017) "Evaluación de la alternativa para la estabilización de taludes mediante la implementación de llantas en la montaña la ponderosa (localidad de ciudad Bolivar, Barrio Bella Flor) " Universidad Distrital Francisco José de Caldas, Bogotá D.C., Colombia.

[23] Sayão A., Sieira A. et al. (2002)."Retaining Walls BuiltScrap Tyre"s. Sociedade Portuguesa de Geotecnia ,91, 39-55. ISBN: 03799522.

[24] Teixeira J. (2012). "Estruturas de suporte de terras executadas com pneus". Instituto superior de engenharia do Porto. Porto, Portugal. 